\title{
Parental Monitoring During Early Adolescence Deters Adolescent Sexual Initiation: Discrete-Time Survival Mixture Analysis
}

\author{
David Y. C. Huang • Debra A. Murphy • \\ Yih-Ing Hser
}

Published online: 21 September 2010

(c) The Author(s) 2010. This article is published with open access at Springerlink.com

\begin{abstract}
We used discrete-time survival mixture modeling to examine 5,305 adolescents from the 1997 National Longitudinal Survey of Youth regarding the impact of parental monitoring during early adolescence (ages 14-16) on initiation of sexual intercourse and problem behavior engagement (ages 14-23). Four distinctive parental-monitoring groups were identified and labeled as "High," "Increasing," "Decreasing," and "Low". About 68\% of adolescents received a high level of parental monitoring from ages 14 to 16 (High), 6 and 9\% respectively exhibited an accelerated (Increasing) and a decelerated trajectory (Decreasing), and $17 \%$ had consistently low parental monitoring (Low). Relative to participants in the Low group, adolescents in the High group delayed sexual initiation by 1.5 years. Males, relative to females, were more likely to have had a low trajectory of parental monitoring, and were more likely to initiate sexual intercourse before age 14. In contrast to White Adolescents, Hispanics and Blacks were less likely to receive High parental monitoring, and had a higher rate of early sexual initiation before age 14 . The study demonstrates the temporal relationship of parental monitoring with adolescent sexual initiation from a longitudinal perspective. An increase of parental monitoring across ages is accompanied with a decrease of sexual risk. The continual high level of parental monitoring from ages 14 to 16 also mitigated the risk of engagement in substance use and delinquent behaviors from ages 14 to 23 .
\end{abstract}

D. Y. C. Huang $(\bowtie) \cdot$ D. A. Murphy · Y.-I. Hser Integrated Substance Abuse Programs, Semel Institute for Neuroscience and Human Behavior, University of California, Los Angeles, 1640 S. Sepulveda Blvd., Suite 200, Los Angeles, CA 90025, USA

e-mail: yhuang@ucla.edu
Keywords Discrete-time survival mixture model . Sexual behavior - Parental monitoring . Alcohol/drug use · Delinquency

\section{Introduction}

Degree of parental monitoring has been linked to adolescent sexual involvement as well as a variety of outcomes (e.g., scholastic achievement, and conduct problems; Otto and Atkinson 1997). Various studies have recognized constructive parental monitoring as the most important and effective factor for mitigating the early onset of sexual activity for adolescents (Coley et al. 2009; Li et al. 2000; Sieverding et al. 2005). In addition to parental monitoring, prior research has examined diverse family and social factors associated with adolescent sexual initation. For instance, socioeconomic status (SES) and parental education were reported to negatively correlate with age of sexual onset (e.g., Johnson and Tyler 2007; Mott et al. 1996; Santelli et al. 2004). Poor school achievement and lack of family connectedness, as well as problem behaviors such as substance use and delinquency were reported as risk factors for early sexual initiation (e.g., Gilliam et al. 2007; Lammers et al. 2000). Church attendance, positive peer norms, and personal beliefs and attitudes regarding postponing sexual initiation (e.g., Gray et al. 2008; O'Donnell et al. 2003) were protective factors that have been associated with delayed adolescent sexual initiation.

While previous research has shown that parental monitoring as well as individual, family, and community characteristics influence adolescent sexual initiation, most of these studies have been cross-sectional, making it difficult to disentangle antecedents from consequences of early sexual intercourse (Crockett et al. 1996). Even in studies 
with longitudinal data, the analytical approaches were not from a longitudinal perspective. Most outcomes were based upon findings from multivariate comparisons (Crockett et al. 1996; O'Donnell et al. 2003), regression modeling (Gray et al. 2008; Mott et al. 1996; Santelli et al. 2004; Stanton et al. 2002), or correlation analysis (Gilliam et al. 2007; Johnson and Tyler 2007), which only evaluated a snapshot effect of a covariate at a specific time point, leaving the dynamic variation of the covariate over time uninvestigated. For example, researchers have applied logistic regression models to demonstrate the significant associations of beliefs and attitudes (Gray et al. 2008) and peer norms and alcohol and drug use (Santelli et al. 2004) with adolescent sexual initiation. Johnson and Tyler (2007), alternatively, applied the path analysis to show that grandmother and mother characteristics were related to the development of adolescent sexual onset.

There have been relatively few attempts to examine the trend effect, or dynamic changes over time, on sexual onset. Some studies applied survival modeling to assess time to sexual initiation (e.g., Capaldi et al. 1996; Lammers et al. 2000; Mitchell et al. 2007; Sieverding et al. 2005). Even though the survival analysis is designed to examine whether and when the first sexual activity occurs and the impact of time-invariant and time-varying covariates on that event (Singer and Willett 2003), most studies primarily focus on examinations of time-invariant covariates, giving relatively limited consideration to the potential impact of changes in covariates over time. For example, Lammers et al. (2000) underscored the importance of family, social and personal characteristics associated with postponement of sexual intercourse using the Cox proportional hazard model; all covariates in their model were single measures and, therefore, lacked repeated measures across time. More recently, Caminis et al. (2007) applied a two-step approach to assess the time-trend effect of a covariate, in which a Hierarchical model was first applied to estimate rate of change over years of covariates (e.g., depression, problem related to substance use); afterwards, the estimated slopes were included in a logistic regression model to examine their impact on adolescent initiation of sexual intercourse. This approach is less efficient statistically because the two models were not estimated simultaneously.

The evidence from cross-sectional analyses generally is limited in establishing the temporality of this association and investigation of underlying pathways (Crockett et al. 1996; Mott et al. 1996; Santelli et al. 2004). For instance, cross-sectional studies may fail to explain the factual role of parental monitoring because it is unable to distinguish between youths continually experiencing low levels of monitoring and those whose monitoring declines as they age (Romer et al. 1999). This limitation also highlights the importance of recognizing the trajectory of risk and protective factors in adolescence and their associations with initiation of sexual intercourse, and of examining these associations with an application of longitudinal statistical analysis.

In light of these issues, the present study seeks to advance prior research by applying a more rigorous statistical methodology-the discrete-time survival mixture analysis-on longitudinal data obtained from a national representative sample of adolescents to examine the temporal association of parental monitoring with adolescent sexual onset. By focusing on the patterns of parental monitoring across years and the ways in which those patterns influence adolescent sexual activities and other problem behaviors over time, this integrated approach offers a broadened organizing framework that potentially allows for characterizing distinctive patterns of parental monitoring during early adolescence, analytically estimating the magnitude of its impact on sexual initiation and other risk behaviors, and examining the contribution of parental monitoring to the persistence or change in risk behaviors during the life span. In particular, the study investigates whether continual parental monitoring during early adolescence impedes the onset of sexual intercourse, and determines whether this protective factor could prevent adolescents from engaging in high-risk sexual behaviors as well as other problem behaviors. The research questions of the study are: (1) What are the distinctive trajectory patterns of parental monitoring during early adolescence?; (2) How do these trajectory patterns differ by gender and ethnicity?; (3) Will different trajectories of parental monitoring result in a delay of youths' sexual initiation?; and (4) What is the long-term impact of parental monitoring on the development of risky sexual behaviors, substance use, and delinquency over time?

\section{Method}

Sample

The 1997 National Longitudinal Survey of Youth (NLSY97; US Department of Labor 2008) is an ongoing survey that longitudinally tracked 8,984 adolescents as they transitioned from school to work. The survey included extensive data on education, employment, sexual activity, delinquency, substance use, family background, and mental health. The NLSY97 comprises a nationally representative sample of youth $(n=6,748)$ and an oversample of Black and Hispanic youth $(n=2,236)$ who were born between 1980 and 1984. Both the eligible youth and one of each youth's parents received hour-long personal interviews in the first survey (1997). Youth have been assessed annually since 1997. Youth data from nine assessment points 
(1997-2005) are examined in the present study. Adolescents were first asked sexual behavior questions at age 14 . In order to appropriately estimate timing of sexual debut, this study includes 5,305 adolescents (2,743 males and 2,562 females) who were 12-14 years old as of December 31,1996 , and had responded to the sexual behavior questions. Of the 5,305 adolescents, $48.3 \%$ were female; $51.8 \%$ were White, 25.8\% African American, 21.4\% Hispanic, and $1.0 \%$ of other ethnic groups. At the end of 1996, their average age was 13 years, with about one-third of the adolescents aged 12, one-third aged 13, and one-third aged 14. At the Wave 1 assessment (1997), about $70 \%$ of these adolescents were in middle school, $20 \%$ in high school, and $10 \%$ in elementary school.

\section{Measures}

The current study utilized data across the nine assessment points (1997-2005) to examine patterns of parental monitoring across ages and their association with the timing of first sexual intercourse for the adolescents. Measures on parental monitoring, sexual activities, alcohol consumption, marijuana use, and delinquency across the nine waves of interviews were included and then temporally rearranged based upon the participant's age at each interview. For instance, a participant's measures on sexual activities for age 14 were obtained from a corresponding wave of interviews at which the respondent was age 14. Participants' demographic characteristics and other contexts, including peer influences, neighborhood safety, school experience, and self expectation were examined from baseline data at Wave 1 (1997). In addition to demographics, the following measures were examined in the study.

\section{Parental Monitoring}

The scale of parental monitoring was comprised of four questions posed to the adolescents about their parents: (1) How much does he/she know about your close friends, that is, who they are?; (2) How much does he/she know about your close friends' parents, that is, who they are?; (3) How much does he/she know about who you are with when you are not at home?; and (4) How much does he/she know about who your teachers are and what you are doing in school? The questions were repeatedly asked for each of the participants' parents at each assessment from Waves 1-4 (1997-2000). Responses were measured on a fivepoint scale. Higher scores indicated greater parental monitoring. The parental monitoring scale was created for residential mother and residential father. The highest level of monitoring from residential mother or father was considered to be the level of parental monitoring that participants received.

\section{Dating and Sexual Activities}

Adolescents aged 14 or older reported their dating and sexual activities at each wave of the survey. Measures included the age they first dated, frequency of dating, age at first sexual intercourse, number of incidents of sexual intercourse, and number of sexual partners in the past 12 months (Wave 1), or since the last interview (subsequent interviews). Starting at Wave 2, questions were asked about whether a condom was used at first intercourse and the number of times a condom was used since the last interview. Age at first sexual intercourse. Timing of first sexual intercourse was identified by examining the responses to questions on "age at first sexual intercourse" and "frequency of sexual intercourse since the last interview" over the nine surveys. Adolescents without sexual debuts by Wave 9 survey were indicated as censored. Sexual risk score. A sexual risk score was computed from three items at each assessment: number of sexual partners, number of incidents of sexual intercourse, and percentage of condom use (computed as times of condom use divided by times of sexual intercourse). Higher scores indicated a higher sexual risk.

\section{Substance Use}

Measures of substance use were constructed from queries about quantity and frequency of three types of substance use: cigarette smoking, alcohol consumption, and marijuana use. The measures were collected at each assessment from Waves 1-9. The number of days of use in the past 30 days was applied to indicate level of substance use.

\section{Delinquency and Arrest}

Delinquency was measured by assessing a broad range of delinquency behaviors and criminal activities: carried a handgun, joined a gang, purposely damaged property, involved in other property crimes, attacked someone, sold illicit drugs, stole a car, or other theft. Any one of the above activities occurring during the observation period (ages 14-23) was considered to be an incident of delinquency. An arrest due to any one of the above activities was also reported.

\section{Peer Influences}

Adolescents estimated the percentage of their peers who got drunk at least once per month, ever used illicit drugs, 
belonged to a gang, planned to go to college, or went to church regularly.

\section{Neighborhood Safety}

Adolescents were asked five questions related to experiences in their neighborhood, e.g., "household was broken into" or "gangs in neighborhood".

\section{School Experience}

Adolescents' experience with their schools and teachers were measured with the statements: (1) Discipline is fair; (2) Disruptions by other students get in the way of my learning; (3) I feel safe at this school; (4) Students are fairly graded; (5) Teachers are good; (6) Teachers are interested in the students; and (7) There is a lot of cheating on tests and assignments.

\section{Self Expectation}

Adolescents' attitudes toward their future were measured with the statements: (1) I hardly ever expect things to go my way; (2) I rarely count on good things happening to me; (3) I'm always optimistic about my future; and (4) In uncertain times, I usually expect the best.

\section{Mental Health}

Mental health was measured with questions on how often in the past month the respondent: (1) had been a nervous person; (2) felt calm and peaceful; (3) felt down or blue; (4) had been a happy person; and (5) felt depressed. The measure was collected at Round 8 (2004). Response options for the school experience, self expectation, and mental health measures range along a 4-point Likert scale. Higher scores indicated better status.

\section{Analyses}

The discrete-time survival mixture modeling (Muthén and Masyn 2005) was applied to test the temporal association of the trajectories of continual parental monitoring from ages 14 to 16 and the development of sexual initiation from ages 14 to 23 for adolescents. The modeling examined the survival curve of sexual initiation in conjunction with the trajectory of parental monitoring simultaneously. In the analysis, levels of parental monitoring from ages 14 to 16 were analyzed by growth mixture curves with a latent class variable to indicate distinctive patterns of parental monitoring trajectories within the sample (Muthén 2004). Timing of adolescent sexual initiation from ages 14 to 23 was modeled by a discrete-time survival curve (Singer and
Willett 2003), which describes the incidence of sexual intercourse across the at-risk time period with estimations of hazard and survival rates. The hazard rate indicates the proportion of at-risk participants (who had not yet had an event) who initiated the event at each age. The survival rate represents the incidence of the event in cumulative fashion; it indicates the percentage of the full sample who survived, or, in this context, remained sexually uninitiated at each age. The two types of curves were linked with an assumption of the impact of distinctive parental monitoring trajectories on timing of sexual initiation. The model also included gender as a time-invariant covariate that affects the latent class of parental monitoring as well as the survival curve of sexual initiation. Figure 1 shows the diagram of the discrete-time survival mixture model applied in this study. The model was conducted using Mplus 5.1 (Muthén and Muthén 2007).

The second part of analyses examined differences among the subsamples of youth belonging to the identified parental monitoring trajectory groups in terms of individual, school, peer, and neighborhood characteristics, as well as other adolescent problem behaviors that were repeatedly assessed across ages. Individual characteristics consisted of ethnicity and mental health status. The school factor was measured by adolescents' ratings of their school experience. Peer influence comprised the percentages of adolescents' peers who ever smoked, used alcohol and illicit drugs, engaged in a gang, cut a class, or went to church regularly. Neighborhood indicators gauged the safety of the adolescent's environment. Differences on these variables among the identified parental-monitoring groups were tested using chisquare for categorical variables and general linear models (SAS Proc GLM) for continuous variables. For variables with repeated measures across ages, including sexual risk, alcohol consumption, and arrest, comparisons were made on trajectories of each variable across ages among the identified parental-monitoring groups.

\section{Results}

"Age to Event" Analysis: Risk of Sexual Initiation Across Ages

Over $85 \%$ of adolescents reported initiation of sexual intercourse by the end of the observation period. The average age of first sexual intercourse was 15.9 years old for males and 16.6 for females. About $17.0 \%$ of males and $7.6 \%$ of females initiated sexual intercourse before age $14\left(X_{(1)}^{2}=107.8, p<.001\right)$. The majority of adolescents become sexually active at ages $14-18$. The rate of sexual initiation across ages presented a concave-shaped trajectory with a peak at age 18; the hazard rate accelerated from ages 
Fig. 1 Diagram of the discretetime survival mixture model with gender as a time-invariant covariate

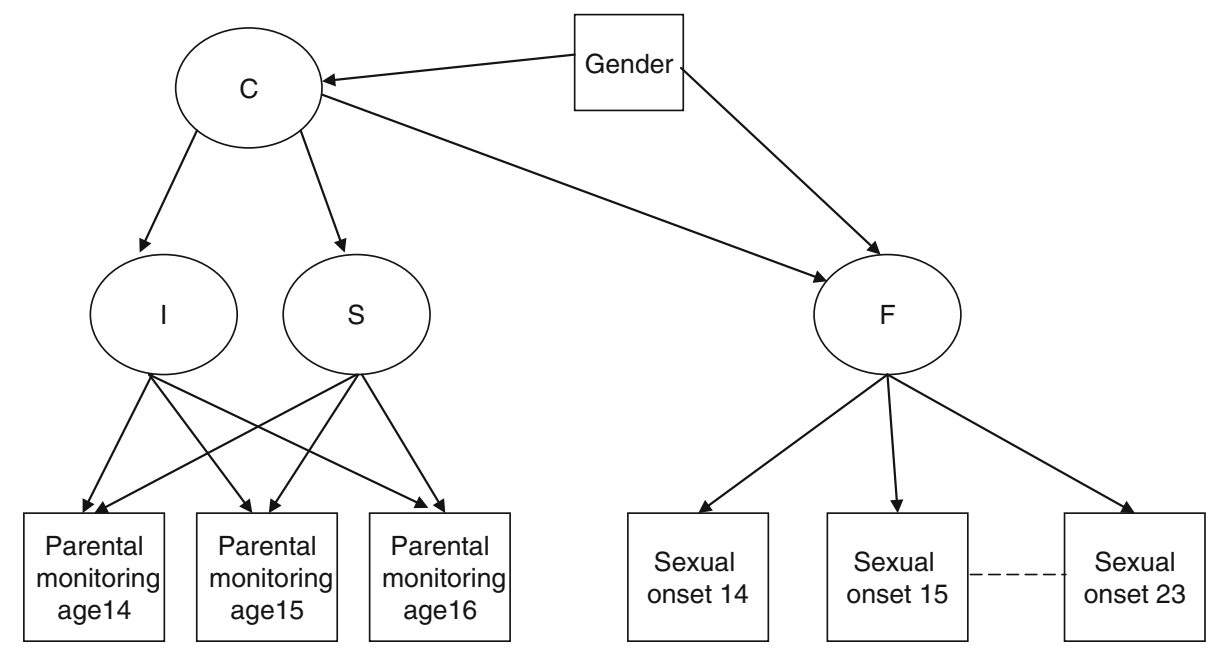

Level of Parental monitoring ages $14 \sim 16$, sexual onset ages 14 23

I, latent intercept factor; S, latent slope factor; C, latent trajectory group

$\mathrm{F}$, latent factor for discrete-time survivals, gender, a time invariant covariate
14 to 18 , reaching the highest level at age 18 , and gradually decelerating afterwards. This indicates that an important time period for intervention programs is between the ages of 14 to 18 . Appropriate intervention programs may decrease sexual risk behavior among adolescents. Males, relative to females, have a higher hazard rate from ages 14 to 18 . However, the hazard rate for males dropped significantly after age 18 because the majority of males had become sexually active at or before age $18 ; 81 \%$ of males and $77 \%$ of females initiated sexual intercourse by age 18 . The hazard rates of sexual initiation are also significantly different by ethnicity; $7 \%$ of Whites, $22 \%$ of Blacks and $13 \%$ of Hispanics initiated sexual intercourse before age $14\left(X_{(3)}^{2}=\right.$ 191.0, $p<.001)$. Of the three ethnic groups, the hazard of sexual initiation across age remained the highest among Blacks, for whom the hazard rates from ages 14 to 18 increased from 21 to $41 \%$. The hazard rates from ages 14 to 18 were 12 to $32 \%$ for Hispanics and 11 to $32 \%$ for Whites.

\section{Trajectories of Parental Monitoring from Ages 14 to 16}

The discrete-time survival mixture model identified four distinctive trajectory groups of parental monitoring-High, Increasing, Decreasing, and Low-in the study sample (Fig. 2a). The High group consists of the majority of participants $(68 \%)$, who received stable high levels of parental monitoring from ages 14 to 16 . Another $6 \%$ of adolescents exhibited an accelerated trajectory; the level of parental monitoring was low at age 14 but significantly accelerated afterwards. Inversely, a decelerated trend in level of parental monitoring was observed among $9 \%$ of participants; level of parental monitoring of these adolescents was high at age 14 but significantly decreased afterwards.
Another $17 \%$ of participants remained with low levels of parental monitoring from ages 14 to 16 . Gender distribution significantly differed among the four groups $\left(X_{(3)}^{2}=92.1\right.$, $p<.001)$. Relative to boys, girls were more likely to be in the High group, indicating that girls generally received a higher level of parental monitoring than boys.

Association of Distinctive Trajectories of Parental Monitoring and the Incidence of Sexual Debut

The discrete-time survival mixture modeling demonstrates the temporal association of the distinctive patterns of parental monitoring with the incidence of sexual activity from ages 14 to 23 , as well as the gender-specific patterns of sexual onset. In contrast to females, males were more likely to initiate sexual activity during this age period (1.12 times, $Z=3.1, p=.002$ ). Relative to participants in the Low group, adolescents in the High group showed a higher survival curve (i.e., were less likely to have had sexual intercourse) across ages. As shown in Fig. 2b, the survival curves for the Increasing and Decreasing groups crossovered at age 16, indicating that an increase in parental monitoring was accompanied by a decrease in risk of sexual initiation. Parental monitoring was related to the rate of sexual initiation throughout the age range studied, with heavily monitored youth exhibiting a lower rate of sexual initiation than less-monitored youth.

The Long-Term Impact of Parental Monitoring on other Adolescent Problem Behaviors

Parental monitoring during early adolescence also has a long-term mitigating impact on adolescent problem 
Fig. 2 Distinctive trajectory groups of level of parental monitoring from ages $14-16$ (Plot $A)$ and survival probability of sexual initiation from ages $14-23$ by the groups (Plot $B$ )
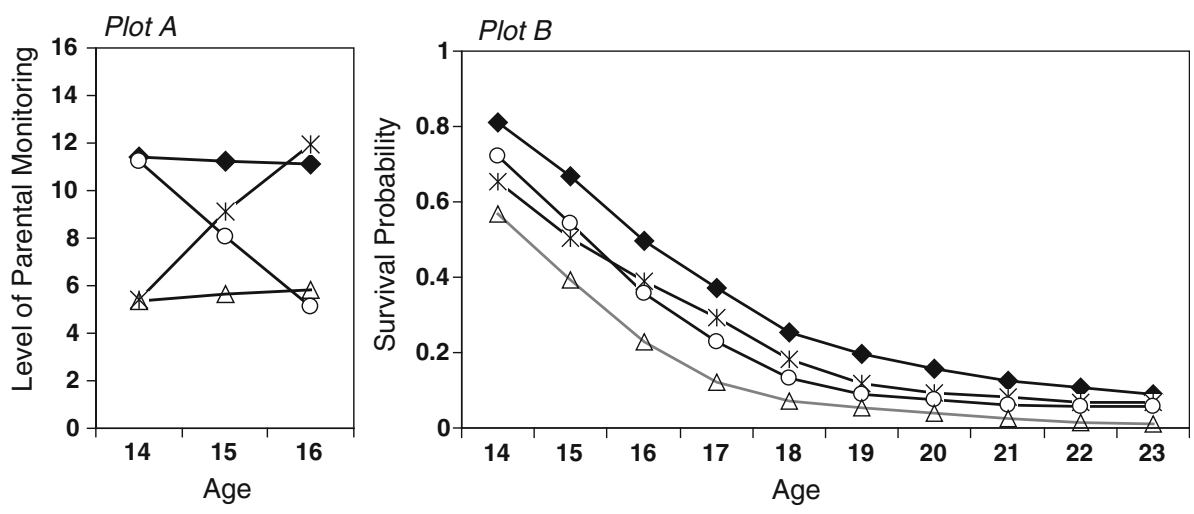

$\multimap$ High $-\nVdash$-Increasing $\rightarrow$ Decreasing $\triangle$ Low behaviors. Adolescents with continually high levels of parental monitoring from ages 14 to 16 showed a relatively low involvement in risky sexual behaviors, alcohol use, and arrest throughout adolescence and young adulthood (ages 14-23). Figure 3a demonstrates distinctive trajectories of sexual risk among adolescents in the four parentalmonitoring groups; adolescents with high and low levels of parental monitoring from ages 14 to 16 , respectively showed the highest and lowest levels of sexual risk score over time. In contrast to the Increasing group, trajectory of sexual risk score for the Decreasing group was lower from ages 14 to 16 , but the two trajectories became indistinguishable after age 16 . Figure $3 \mathrm{~b}$ shows that trajectory of alcohol use for the Increasing group was higher than that for the High group before age 16 but became lower than that for the High group after age 16, indicating that an increase of parental monitoring in early adolescence alleviates level of alcohol use in adolescence and young adulthood. Additionally, incidents of arrest significantly correspond to level of parental monitoring; adolescents with a continual high level of parental monitoring from ages 14 to 16 exhibited a relatively low arrest rate through adolescence and young adulthood (Fig. 3c).

Individual, Peer, and Neighborhood Characteristics by the Parental-Monitoring Groups

Table 1 compares individual, peer, and neighborhood characteristics by the four distinctive parental-monitoring groups. Of the four parental-monitoring groups, adolescents in the High group showed the lowest incidence of early problem behaviors. In addition to adolescents in the Low group, adolescents in the Increasing group also showed higher incidence of early problem behaviors by age 14 , suggesting that early problem behavior consistently corresponds to level of parental monitoring. Parental monitoring also significantly prevents adolescents from negative peer influence. Compared to adolescents in the
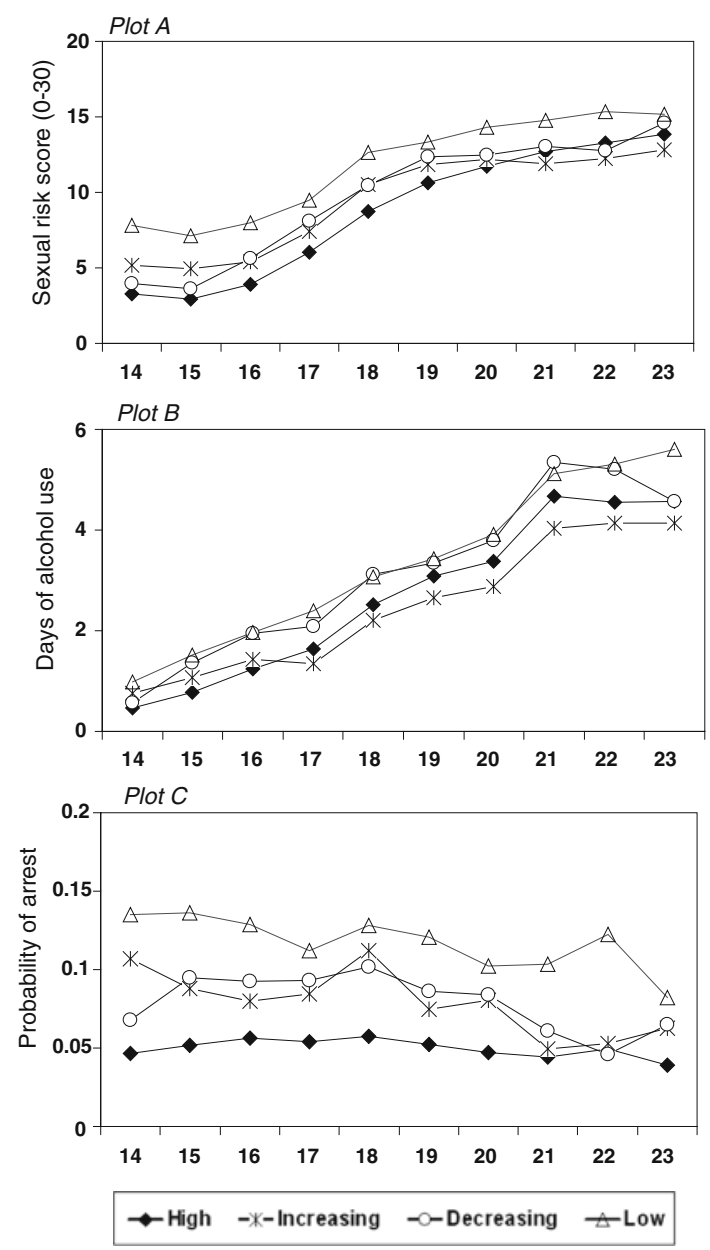

Fig. 3 Sexual risk score (Plot A), Days of alcohol consumption (Plot B), and probability of arrest (Plot C) from age 14-23 by the parental-monitoring groups

Low group, adolescents in the High group had a significantly lower percentage of peers who ever smoked, consumed alcohol, used drugs, belonged to a gang, or cut a class. Level of parental monitoring was also positively associated with neighborhood safety. Adolescents with a 
Table 1 Individual, peer, and neighborhood characteristics by the four parental-monitoring groups

\begin{tabular}{|c|c|c|c|c|c|}
\hline Variables & $\begin{array}{l}\text { High } \\
(n=3,781)\end{array}$ & $\begin{array}{l}\text { Increasing } \\
(n=262)\end{array}$ & $\begin{array}{l}\text { Decreasing } \\
(n=443)\end{array}$ & $\begin{array}{l}\text { Low } \\
(n=819)\end{array}$ & $\begin{array}{l}\text { Total } \\
(n=5,305)\end{array}$ \\
\hline \multicolumn{6}{|l|}{ Gender* } \\
\hline Males & 48.5 & 42.8 & 60.5 & 64.6 & 51.7 \\
\hline Females & 51.5 & 57.3 & 39.5 & 35.4 & 48.3 \\
\hline \multicolumn{6}{|l|}{ Ethnicity* } \\
\hline Whites & 54.5 & 38.9 & 51.9 & 43.7 & 51.9 \\
\hline African Americans & 24.1 & 35.5 & 24.8 & 31.1 & 25.8 \\
\hline Hispanics & 20.5 & 24.4 & 22.4 & 23.9 & 21.4 \\
\hline Others & 0.9 & 1.2 & 0.9 & 1.2 & 0.9 \\
\hline \multicolumn{6}{|c|}{ Early engagement in problem behaviors by age 14} \\
\hline Had sex* & 18.8 & 34.7 & 28.0 & 43.1 & 24.1 \\
\hline Smoked cigarettes* & 12.4 & 26.7 & 16.0 & 26.7 & 15.6 \\
\hline Drank alcohol* & 14.7 & 22.9 & 14.9 & 26.8 & 17.0 \\
\hline Used marijuana* & 5.0 & 12.6 & 6.1 & 12.0 & 6.5 \\
\hline Involved in gang* & 2.6 & 9.2 & 4.3 & 9.8 & 4.1 \\
\hline Arrested* & 4.6 & 10.7 & 6.8 & 13.5 & 6.5 \\
\hline \multicolumn{6}{|c|}{ Peer influence: percentage of peers who } \\
\hline Smoked cigarettes* & 61.1 & 66.2 & 59.4 & 70.8 & 62.7 \\
\hline Drunk at least once a month* & 37.0 & 42.9 & 31.6 & 48.9 & 61.3 \\
\hline Ever used drugs* & 46.3 & 44.7 & 46.1 & 61.9 & 49.1 \\
\hline Belonged to a gang* & 39.0 & 40.7 & 31.1 & 45.1 & 32.2 \\
\hline Cut class/school* & 55.7 & 68.2 & 60.5 & 72.8 & 59.4 \\
\hline Went to church regularly* & 88.6 & 82.0 & 87.2 & 82.9 & 87.3 \\
\hline Plan to go to college* & 96.6 & 96.1 & 95.6 & 93.3 & 96.0 \\
\hline \multicolumn{6}{|l|}{ Neighborhood safety } \\
\hline \multicolumn{6}{|l|}{ Residence area* } \\
\hline Rural & 24.0 & 21.8 & 19.2 & 18.2 & 22.6 \\
\hline Urban & 71.7 & 74.8 & 77.4 & 77.5 & 73.2 \\
\hline Unknown & 4.4 & 3.4 & 3.4 & 4.3 & 4.2 \\
\hline Hear a gunshot during a day* & 19.2 & 28.1 & 22.2 & 29.7 & 21.4 \\
\hline Gangs in neighborhood* & 40.8 & 53.3 & 45.5 & 54.3 & 43.9 \\
\hline Household was broken into* & 14.0 & 16.5 & 16.9 & 22.0 & 15.6 \\
\hline Victim of bullying* & 18.2 & 20.5 & 17.3 & 27.0 & 19.6 \\
\hline See someone shot with gun* & 9.0 & 13.5 & 13.7 & 18.6 & 11.1 \\
\hline School experience $(7-28)^{*}$ & $20.3(2.9)$ & $18.9(3.1)$ & $19.9(3.2)$ & $18.8(2.9)$ & $19.9(3.0)$ \\
\hline Self expectation $(4-16)^{*}$ & $11.5(1.9)$ & $10.9(1.7)$ & $11.2(1.9)$ & $10.7(1.8)$ & $11.3(1.9)$ \\
\hline Mental health 2004, (5-20)* & $15.6(2.4)$ & $15.4(2.5)$ & $15.2(2.6)$ & $15.1(2.7)$ & $15.5(2.5)$ \\
\hline
\end{tabular}

$* p<0.01$

high level of parental monitoring were more likely to live in a much safer neighborhood. Relative to the Low group, a lower proportion of adolescents in the High groups encountered an unsafe event in their neighborhood, including hearing gunshots, observing gang activity in the neighborhood, having their house broken into, being a victim of bullying, or seeing someone shot with a gun. Adolescents in the High parental monitoring group, compared to their counterparts, had better school experiences, higher future expectation, and better mental health.

\section{Discussion}

The strengths of this study include the use of a national representative sample of youth (The NLSY97), and the application of the discrete-time survival mixture modeling approach for examination of the concurrent and prospective relationships of parental monitoring with adolescent sexual activity and other problem behaviors. The NLSY97 data, which comprises repeated assessments of parental monitoring, substance use and delinquency, as well as 
timing of sexual initiation, provide an excellent opportunity to assess the long-term impact of parental monitoring on adolescent sexual activities from a framework of multivariate longitudinal analysis. The discrete-time survival mixture model provides an innovate approach to examine the temporal association of parental monitoring with adolescent sexual onset. This modeling approach identifies distinctive patterns of level of parental monitoring in adolescence, accommodates varying timing of first sexual intercourse, and simultaneously estimates the temporal association of parental monitoring on sexual initiation. Several findings were noteworthy. First, continual parental monitoring significantly postponed adolescent sexual initiation, confirming previous cross-sectional results. Adolescents with continual parental monitoring over time were less likely to initiate sex early than participants whose parents either never monitored them or discontinued monitoring them as they aged. Second, the level of parental monitoring was also correlated with the risk of problem behavior engagement. Adolescents with a higher level of parental monitoring, compared to their counterparts, showed lower sexual risk; lower levels of cigarette smoking, alcohol consumption, and marijuana use; and lower risk of gang involvement and arrest. These results suggest that constructive parental monitoring effectively delays sexual initiation and has additional value in mitigation of risk of substance use and delinquency.

Our analyses showed that adolescents in the Low parental-monitoring group, on average, had the earliest sexual initiation at age of 14.7 , compared to the ages of 16.2, 15.4 and 15.3, for the High, Increasing and Decreasing groups, respectively. This finding provides additional empirical evidence of the impact of parental monitoring and has important implications from the perspective of child development. High parental monitoring, in contrast to Low parental monitoring, results in an average of 1.5 years of postponement of adolescent sexual initiation. That 18-months age difference is critically important for adolescents because dramatic cognitive and social developments take place during each year of adolescence (Steinberg 2005). The increasing cognitive development provides adolescents new levels of social awareness and improves their capacity to make sound judgments. An additional 18 months of development is particularly helpful for adolescents to develop better coping skills and improve their abilities in decision-making and problem-solving, including negotiation skills for safer sex, and they may consequently avoid coercive and/or unprotected sex, risky sexual activities, and problem behaviors in subsequent years.

The distinctive parental-monitoring trajectories indicate the dynamic nature of parental monitoring in adolescence. The subgroups that deserve further discussion are adolescents in the Increasing and Decreasing groups because their developmental trajectories considerably demonstrate the concurrent and prospective impact of parental monitoring on sexual initiation and problem behavior involvement in adolescence. Adolescents in the former and the latter groups respectively showed a consistent acceleration and a consistent deceleration on level of parental monitoring from ages 14 to 16 . An increase (or decrease) in level of parental monitoring resulted in a concurrent decrease (or increase) on risk of sexual initiation and on probability of problem behavior engagement from ages 14 to 16 . In addition to the concurrent effect, the impact remained consistent through the subsequent years of the observation period (ages 17-23). These empirical findings imply that it is never too late for parents to increase their monitoring of adolescents. It is still effective for parents who had not monitored early on to begin better parental monitoring. Timely parental monitoring throughout adolescence is generally worthwhile in preventing adverse consequences of adolescent problem behaviors. The findings also suggest the possible superiority of family-based interventions, as opposed to interventions that focus only on adolescents. Parents need to strengthen their monitoring capabilities and develop appropriate skills to establish constructive communication and mutual-trust relationships with their children, especially as their children transition to early/middle adolescence, and then to late adolescence.

The results from our longitudinal analysis also showed gender-specific and ethnic-specific patterns of timing of sexual debut for adolescents. Consistent with findings from cross-sectional analyses (e.g., Mott et al. 1996; O'Donnell et al. 2003), males, relative to females, were more likely to have had a low trajectory of parental monitoring, and were more likely to initiate sexual intercourse before age 14 . In contrast to White adolescents, Hispanics and Blacks were less likely to have had a High parental monitoring trajectory through adolescence, and a higher percentage of Black and Hispanic adolescents had their first sexual intercourse before age 14. The ethnic differences on trajectories of parental monitoring consistently corresponded to ethnicspecific patterns of early sexual initiation.

In addition to prior evidence (e.g., O'Donnell et al. 2003; Santelli et al. 2004) that demonstrated a direct association between norms, personal attitudes and peer influence on sexual initiation, this study underscores the linkage of distinctive parental-monitoring trajectories with individual, peer, and neighborhood characteristics, which may indirectly delay sexual initiation. Compared to their counterparts, adolescents in the High parental monitoring group had better school experiences and higher future expectations, which all lead to deferment of sexual initiation. On the other hand, adolescents in the Low parental-monitoring 
group, relative to their counterparts, were more likely to engage in deviant behaviors and to encounter an unsafe event in their neighborhood such as gang activity or hearing gun shots; the adolescents also reported higher percentages of their peers ever smoking, using drugs, and belonging to a gang. The interrelationships of parental monitoring with personal values, peer influence, and perceived environment reflect the complexity of possible etiologic mechanisms of adolescent sexual behaviors and indicate a need for an integrated intervention program with a combination of efforts from family, school, and community. Development of a tight family connectedness, reduction of the chance of exposure to negative peer influences, and improvement in the safety of the community could lead to reducing early sexual onset and problem behaviors among adolescents.

In sum, development in adolescence is a complex course with versatile paths and multiple consequences (Donenberg et al. 2006). Latent class growth mixture modeling, in conjunction with discrete-time survival analysis, allows us to critically examine the relationships between two developmental processes, parental monitoring and sexual onset. This study contributes to previous literature with an examination of a time-trend effect of parental monitoring, instead of a snapshot effect at a signal time point, on sexual initiation. Parental monitoring in the preadolescent years (age 14-16) can affect the developmental course of sexual risk behaviors through the subsequent years of adolescence. The most important implication of the study is that an effective intervention program should be designed to influence both adolescents and their parents. An integrated intervention with appropriate involvement and support from parents will have better long-term effects than efforts that target adolescents alone.

\section{Limitations}

There are several potential limitations in the current study. First, sample attribution across multiple waves of followup may undermine the reliability of estimations of trajectories of parental monitoring, sexual risk, and problem behaviors across ages. This may also limit the generalizability of the research findings. However, subjects with missing data at a few time points were not dropped from the discrete-time survival mixture model analysis. With an assumption of missing at random (MAR), trajectories were estimated by using all available observations. The influence of attrition is relatively minor. Second, measures of parental monitoring were only collected at the first four waves of the survey. Insufficient data limit our further examinations of the extent of parental monitoring on risky behavior engagement during late adolescence. Third, measures of parental monitoring, sexual activity, and problem behaviors primarily relied on the self-report of adolescents. Incidences of problem behaviors based upon self-report may be underestimated. However, sensitive questions were asked by the computer-assisted personal interviews (CAPI) system. This arrangement would lower the likelihood of a false report and improve the reliability and validity of responses.

Acknowledgments This study is supported in part under Grant 1R03MH084434-01A1 from the National Institute of Mental Health and by the University of California, Los Angeles, Center for Advancing Longitudinal Drug Abuse Research (CALDAR) under Grant P30DA016383 from the National Institute on Drug Abuse (NIDA). Dr. Hser is also supported by a Senior Scientist award from NIDA (K05DA017648).

Open Access This article is distributed under the terms of the Creative Commons Attribution Noncommercial License which permits any noncommercial use, distribution, and reproduction in any medium, provided the original author(s) and source are credited.

\section{References}

Caminis, A., Henrich, C., Ruchkin, V., Schwab-Stone, M., \& Martin, A. (2007). Psychosocial predictors of sexual initiation and highrisk sexual behaviors in early adolescence. Child and Adolescent Psychiatry and Mental Health, 1, 1-14.

Capaldi, D. M., Crosby, L., \& Stoolmiller, M. (1996). Predicting the timing of first sexual intercourse for at-risk adolescent males. Child Development, 67, 344-359.

Coley, R. L., Votruba-Drzal, E., \& Schindler, H. (2009). Fathers' and mothers' parenting predicting and responding to adolescent sexual risk behaviors. Child Development, 80, 808-827.

Crockett, L. J., Bingham, C. R., Chopak, J. S., \& Vicary, J. R. (1996). Timing of first sexual intercourse: The role of social control, social learning, and problem behavior. Journal of Youth and Adolescence, 25, 89-111.

Donenberg, G. R., Emerson, E., Bryant, F. B., \& King, S. (2006). Does substance use moderate the effects of parents and peers on risky sexual behaviour? AIDS Care, 18, 194-200.

Gilliam, M. L., Berlin, A., Kozloski, M., Hernandez, M., \& Grundy, M. (2007). Interpersonal and personal factors influencing sexual debut among Mexican-American young women in the United States. Journal of Adolescent Health, 41, 495-503.

Gray, S. H., Austin, S. B., Huang, B., Frazier, A. L., Field, A. E., \& Kahn, J. A. (2008). Predicting sexual initiation in a prospective cohort study of adolescents. Archives of Pediatrics and Adolescent Medicine, 162, 55-59.

Johnson, K., \& Tyler, K. (2007). Adolescent sexual onset: An intergenerational analysis. Journal of Youth and Adolescence, 36, 939-949.

Lammers, C., Ireland, M., Resnick, M., \& Blum, R. (2000). Influences on adolescents' decision to postpone onset of sexual intercourse: A survival analysis of virginity among youths aged 13 to 18 years. Journal of Adolescent Health, 26, 42-48.

Li, X., Stanton, B., \& Feigelman, S. (2000). Impact of perceived parental monitoring on adolescent risk behavior over 4 years. Journal of Adolescent Health, 27, 49-56.

Mitchell, C. M., Whitesell, N. R., Spicer, P., Beals, J., Kaufman, C. E., \& The pathways of choice and healthy ways project team. (2007). Cumulative risk for early sexual initiation among American Indian youth: A discrete-time survival analysis. Journal of Research on Adolescence, 17, 387-412. 
Mott, F. L., Fondell, M. M., Hu, P. N., Kowaleski-Jones, L., \& Menaghan, E. G. (1996). The determinants of first sex by age 14 in a high-risk adolescent population. Family Planning Perspectives, 28, 13-18.

Muthén, B. (2004). Latent variable analysis: Growth mixture modeling and related techniques for longitudinal data. In D. Kaplan (Ed.), Handbook of quantitative methodology for the social sciences (pp. 345-368). Newbury Park, CA: Sage Publications.

Muthén, B., \& Masyn, K. (2005). Discrete-time survival mixture analysis. Journal of Educational and Behavioral Statistics, 30 , 27-58.

Muthén, L. K., \& Muthén, B. (2007). Mplus user's guide (Third edition ed.). Los Angeles, CA: Muthén \& Muthén.

O’Donnell, L., Myint-U, A., O’Donnell, C. R., \& Stueve, A. (2003). Long-term influence of sexual norms and attitudes on timing of sexual initiation among urban minority youth. Journal of School Health, 73, 68-75.

Otto, L. B., \& Atkinson, M. P. (1997). Parental involvement and adolescent development. Journal of Adolescent Research, 12, $68-89$.

Romer, D., Stanton, B., Galbraith, J., Feigelman, S., Black, M. M., \& $\mathrm{Li}$, X. (1999). Parental influence on adolescent sexual behavior in high-poverty settings. Archives of Pediatrics and Adolescent Medicine, 153, 1055-1062.

Santelli, J. S., Kaiser, J., Hirsch, L., Radosh, A., Simkin, L., \& Middlestadt, S. (2004). Initiation of sexual intercourse among middle school adolescents: The influence of psychosocial factors. Journal of Adolescent Health, 34, 200-208.

Sieverding, J. A., Adler, N., Witt, S., \& Ellen, J. (2005). The influence of parental monitoring on adolescent sexual initiation. Archives of Pediatrics and Adolescent Medicine, 159, 724-729.

Singer, J. D., \& Willett, J. B. (2003). Applied longitudinal data analysis: Modeling change and event occurrence. New York: Oxford University Press, Inc.

Stanton, B., Li, X., Pack, R., Cottrell, L., Harris, C., \& Burns, J. M. (2002). Longitudinal influence of perceptions of peer and parental factors on African American adolescent risk involvement. Journal of Urban Health: Bulletin of the New York Academy of Medicine, 79, 536-548.

Steinberg, L. (2005). Cognitive and affective development in adolescence. TRENDS in Cognitive Sciences, 9, 69-74.

US Department of Labor, Bureau of Labor Statistics. (2008). The NLSY97. Retrieved March 14, 2008, from http://www.bls. gov/nls/nlsy97.htm. 